\title{
The clinical relevance of formal thought disorder in the early stages of psychosis: results from the PRONIA study
}

\author{
Oemer Faruk Oeztuerk ${ }^{1,2,3}$ (D) Alessandro Pigoni ${ }^{4} \cdot$ Julian Wenzel $^{5} \cdot$ Shalaila S. Haas $^{6} \cdot$ David Popovic $^{1,2} \cdot$ Anne Ruef $^{1}$. \\ Dominic B. Dwyer ${ }^{1} \cdot$ Lana Kambeitz-Ilankovic $^{5} \cdot$ Stephan Ruhrmann $^{5} \cdot$ Katharine Chisholm $^{7} \cdot$ Paris Lalousis $^{8}$. \\ Sian Lowri Griffiths ${ }^{8}$. Theresa Lichtenstein ${ }^{5} \cdot$ Marlene Rosen $^{5}$. Joseph Kambeitz ${ }^{5}$. Frauke Schultze-Lutter ${ }^{9}$. \\ Peter Liddle ${ }^{10} \cdot$ Rachel Upthegrove $^{7,8} \cdot$ Raimo K. R. Salokangas $^{11}$. Christos Pantelis ${ }^{12,13}$. Eva Meisenzahl ${ }^{9}$. \\ Stephen J. Wood ${ }^{14,15,16}$. Paolo Brambilla ${ }^{17}$. Stefan Borgwardt ${ }^{18}$. Peter Falkai ${ }^{1}$. Linda A. Antonucci ${ }^{1,19,20}$. \\ Nikolaos Koutsouleris ${ }^{1,3,21}$. the PRONIA Consortium
}

Received: 1 May 2021 / Accepted: 27 August 2021 / Published online: 17 September 2021

(c) The Author(s) 2021

\begin{abstract}
Background Formal thought disorder (FTD) has been associated with more severe illness courses and functional deficits in patients with psychotic disorders. However, it remains unclear whether the presence of FTD characterises a specific subgroup of patients showing more prominent illness severity, neurocognitive and functional impairments. This study aimed to identify stable and generalizable FTD-subgroups of patients with recent-onset psychosis (ROP) by applying a comprehensive datadriven clustering approach and to test the validity of these subgroups by assessing associations between this FTD-related stratification, social and occupational functioning, and neurocognition.

Methods 279 patients with ROP were recruited as part of the multi-site European PRONIA study (Personalised Prognostic Tools for Early Psychosis Management; www.pronia.eu). Five FTD-related symptoms (conceptual disorganization, poverty of content of speech, difficulty in abstract thinking, increased latency of response and poverty of speech) were assessed with Positive and Negative Symptom Scale (PANSS) and the Scale for the Assessment of Negative Symptoms (SANS).

Results The results with two patient subgroups showing different levels of FTD were the most stable and generalizable clustering solution (predicted clustering strength value $=0.86$ ). FTD-High subgroup had lower scores in social $\left(p_{\mathrm{fdr}}<0.001\right)$ and role $\left(p_{\mathrm{fdr}}<0.001\right)$ functioning, as well as worse neurocognitive performance in semantic $\left(p_{\mathrm{fdr}}<0.001\right)$ and phonological verbal fluency $\left(p_{\mathrm{fdr}}<0.001\right)$, short-term verbal memory $\left(p_{\mathrm{fdr}}=0.002\right)$ and abstract thinking $\left(p_{\mathrm{fdr}}=0.010\right)$, in comparison to FTD-Low group.

Conclusions Clustering techniques allowed us to identify patients with more pronounced FTD showing more severe deficits in functioning and neurocognition, thus suggesting that FTD may be a relevant marker of illness severity in the early psychosis pathway.
\end{abstract}

Keywords Formal thought disorder $\cdot$ Early psychosis $\cdot$ Clustering $\cdot$ Functioning $\cdot$ Neurocognition

Linda A. Antonucci and Nikolaos Koutsouleris have contributed equally to the work.

Oemer Faruk Oeztuerk

oemer.oeztuerk@med.uni-muenchen.de

Extended author information available on the last page of the article

\section{Introduction}

Psychotic disorders are closely linked with neurocognitive and functional impairments $[17,49]$ that frequently precede disease onset and persist after remission of the acute illness $[22,37]$. Formal thought disorder (FTD) is a multifaceted construct of disturbances in thought, communication and language, such as loosening of associations, blocking, semantic and phonemic paraphasia [28, 42]. Previous literature revealed that FTD is not only a core feature of psychosis but that it is also associated with adverse social and 
functional outcomes in psychotic patients [3, 21, 25, 27, 28]. More specifically, FTD is associated with an increased (re-) hospitalization rate [44], unemployment risk [34], reduced quality of life [48] and adjustment abilities indexed by occupational functioning and self-support [34]. Harrow et al. [20] highlighted that patients with schizophrenia experiencing enduring FTD after the acute phase of psychosis showed lower occupational functioning levels and higher relapse/rehospitalisation rates. Moreover, thought and communication disturbances in youth at clinical high risk for psychosis were associated with reduced social and occupational functioning outcomes and a higher risk for transition to the established disease [46]. Furthermore, cognitive basic symptoms including subjective thought blockage, interference and pressure as well as disturbances of abstract thinking and expressive and receptive speech that might be regarded as subclinical presentations of observable FTD have been demonstrated to predict subsequent psychosis [45]. In summary, these findings may point towards FTD playing an important role in explaining the behavioural, psychopathological and functional heterogeneity of disease manifestations in both early and prodromal phases of psychosis. So far, this aspect has remained under-investigated, $[42,43]$ as research on clinical markers of psychosis has focused rather on positive and negative symptoms as well as on cognitive phenotypes of the disorders [44]. However, this traditional approach may not fully capture the clinical heterogeneity of psychosis in terms of both disease course and severity [21, 24, 27, 42]. FTD may represent a clinical fingerprint of disease severity [42] as it encompasses observable speech-related, and cognitive impairments of psychosis. As findings from a recent systematic review indicated, FTD - especially disorganization-might have early diagnostic and prognostic relevance in the early stages of psychosis [39]. The main findings of this systematic review showed that FTD severity predicted poor social functioning, unemployment, relapses, rehospitalisations, as well as correlations between attentional deficits, executive functions and FTD severity, and highlighted the predictive potential of executive dysfunctions for sustained FTD. Therefore, FTD stratification could help clinician to detect a subgroup of patients at risk of developing poor disease outcomes, who may need early preventive interventions targeting specifically FTD-related deficits.

Recently, there has been great interest in machine learning and pattern recognition techniques, which have shown to be promising tools for addressing clinical heterogeneity in psychiatric disorders [7, 15, 35]. Among these, unsupervised clustering algorithms allow us to explore the subgroup structure of psychopathological phenomena in a quantitative and potentially unbiased way [23]. Using unsupervised machine learning techniques to investigate the role of FTD role in the heterogeneity of psychosis phenotypes would allow (i) identifying more homogeneous clinical subgroups experiencing differential illness manifestations, and (ii) exploring the interdependence of possible FTD clusters with other phenotypic expressions of psychosis.

Thus, this study aims for the first time (1) to evaluate whether it is possible to identify robust subtypes of patients with recent-onset psychosis (ROP) that are characterized by distinct FTD patterns, (2) to investigate whether this FTDrelated stratification is associated with clinical (i.e., the Global Social (GF-Social) and Role (GF-Role) Functioning), and neurocognitive (i.e., Wechsler Adult Intelligence Scale (WAIS-III; premorbid verbal intelligence), Phonological and Semantic Verbal Fluency (VF-P \& S), and Auditory Forward and Backward Digit Span (ADS-Forward and Backward) phenotypes at an early stage of the disease, and (3) to explore the potential associations between FTD-related symptoms, functioning and neurocognition.

\section{Methods}

The PRONIA (Personalised Prognostic Tool for Early Psychosis Management) study recruited patients into the recentonset psychosis (ROP) study group if, i) they fulfilled the DSM-IV-TR criteria for affective and non-affective psychotic episode lifetime, ii) the psychotic episode was present within the past 3 months, and iii) the onset of psychosis occurred within the past 24 months. Exclusion criteria were treatments with antipsychotic medication for longer than 90 days (cumulative number of days) at or above minimum dosage of the 1st episode psychosis range of (DGPPN) S3 guideline. [16] General inclusion and exclusion criteria are detailed in the Supplementary Sect. 1. Based on these criteria, we were able to analyse clinical and neurocognitive data from 279 individuals experiencing a ROP between 15 and 40 years of age (Table 1). Patients were recruited by the PRONIA Consortium between January 2014 and December 2017 at ten sites across five countries (Table 1). All adult participants gave their written informed consent prior to study inclusion. Participants younger than 18 years provided written, informed assent, and their caregivers written, informed consent before being enrolled in the study.

1) Psychopathological assessment of the severity of formal thought disorder

The FTD severity has been assessed with different scales $[1,8,14]$ since Kraepelin and Bleuer postulated the importance of earlier manifestation of this clinical phenomenon in an evolving psychosis [21]. The Thought and Language disorder (TALD) scale from Kircher et al. [28] is a validated instrument for assessing FTD. Indeed, it allows clinicians to examine the multifaceted nature of FTD and distinguish 
Table 1 Study-associated sociodemographic

\begin{tabular}{llllr}
\hline & \multicolumn{3}{l}{$\begin{array}{l}\text { Formal thought disorder related } \\
\text { symptom severity }\end{array}$} \\
\cline { 2 - 5 } & Low & High & $\chi^{2}$ & $p$ value \\
\hline Age, median & 24 & 23 & & 0.011 \\
Female, No. (\%) & $91(44)$ & $28(37)$ & 0.7053 & 0.401 \\
Education year, median & 14 & 12 & & $<0.001$ \\
Participants per site, No & & & & \\
The Ludwig-Maximilian- & 76 & 20 & 16.452 & 0.058 \\
University Munich & & & & \\
The University of Cologne & 30 & 15 & & \\
The University of Münster & 6 & 4 & & \\
The University of Düsseldorf & 2 & 3 & & \\
The University of Basel & 18 & 6 & & \\
The University of Turku & 33 & 7 & & \\
The University of Milan & 15 & 10 & & \\
The University of Udine & 8 & 3 & \\
The University of Bari & 1 & 3 & \\
The University of Birmingham & 16 & 3 & \\
\hline
\end{tabular}

positive and negative thought disorder with subjective and objective components. We operationalized only observed positive and negative FTD with items from the Positive and Negative Symptom Scale (PANSS) [26] and the Scale for the Assessment of Negative Symptoms (SANS) [2] with a psychopathological orientation on the Thought and Language disorder (TALD) scale from Kircher et al. [28]

More in detail, observed positive FTD was assessed through:

- conceptual disorganization (PANSS P2) item, reflecting the following psychopathological alterations listed in the TALD scale; tangentiality, circumstantiality, derailment, dissociation of thinking, cross talk, and logorrhoea [26, 28].

- poverty of content speech (SANS 10) item is included in the TALD scale [2, 28]

On the other hands, observed negative FTD was assessed through:

- the difficulty in abstract thinking (PANSS N5) item is called as concretism in the TALD scale, that reflects the same psychopathological alteration [26, 28].

- increased latency of response (SANS 12) item is called as slowed thinking in the TALD scale that also reflects the same psychopathological alteration $[2,28]$.

- poverty of speech (SANS 9) item is included in the TALD scale $[2,28]$.

These five FTD-related symptoms were used as features to cluster patients with ROP into FTD subgroups. Notably, PANSS N6 item (i.e., "Lack of spontaneity and flow of conversation") and SANS 11 item (i.e., "Blocking") individual scores reflecting following TALD scale subjective negative items: "Poverty of thought", "Dysfunction of thought initiative", "Intentionality and expressive speech dysfunction" and "Inhibited thinking", were not included as a feature on purpose in the present study to avoid redundancy between objective and subjective FTD assessments [28, 33].

Trained clinicians assessed psychopathology of each participant and interrater reliability tests were performed regularly to calibrate PANSS [Intraclass Correlation $($ ICC $)=0.79$ ] across study sites. Interrater reliability test for SANS is not available.

2) Clustering FTD subgroups based on psychopathological patterns

Our first aim was to identify a stable and generalizable ROP patient stratification based on distinct patterns of FTD identified by means of data-driven unsupervised machine learning techniques. After scaling the data (Supplementary Sect. 2), we applied the following steps to investigate and compare alternative FTD subgroup solutions.

\section{2a) Validity and stability of clustering methods}

First, we used the ClValid [6] package to assess three clustering algorithms: (i) k-means, (ii) hierarchical clustering, and (iii) partitioning around medoids (PAM) with the number of clusters ranging from 2 to 10. ClValid [6] compares solutions from several clustering algorithms while the numbers of clusters vary. This allows researchers to choose the optimal algorithm and number of clusters with a majority rule based on a battery of internal validity and stability measures (for a full description, see [6]). We tested average and ward linkage for the hierarchical clustering due to previous simulation study from Walesiak and Dudek [54] reporting them as the best linkages for ordinal data. The average linkage considers the distance between two clusters as the average distance between each point in one cluster to every point in the other cluster, whereas ward linkage is a method that minimizes the error sum of squares between the clusters over all the variables. We selected the optimal algorithms and numbers of clusters by applying a majority rule among computed internal validity and stability measures (Supplementary Sect. 3).

Second, we retested the winner algorithms determined with majority rule in the first step with the NbClust [11] package for the optimal number of clusters (Supplementary Sect. 3). Many indices have been reported previously to decide for a valid clustering solution in a given dataset. NbClust provides an automatized scan through 26 validity indices such Calinski-Harabasz $(\mathrm{CH})$ Index [9], Davies-Bouldin (DB) Index [13], Silhouette Index [46] and 
reports the optimal number of clusters for a given clustering algorithm with majority rule. Following the first step, we tested the average and ward linkage for the hierarchical clustering algorithm, which was one of the winner algorithms (Supplementary Sect. 3).

2b) Stability and generalizability of the optimal cluster solutions

Given that the first and second steps resulted in more than one optimal solution, namely k-means and hierarchical algorithms (Supplementary Sect. 3), we inspected the solutions from the hierarchical clustering for their clinical validity and robustness with the figure for silhouette width as well as the cophenetic correlation in a third step (Supplementary Sect. 4).

Thereafter, we examined the stability and generalizability of k-means algorithm solution selected based on the previous steps (Supplementary Sect. 5 and 6). To exclude possible package or function-specific effects, we retested the robustness of the k-means-based clustering solution with the R package ClusterStability [31]. This package allows screening popular validity measures and provides researchers with a global stability (ST) index and an individual STindex ranging from 0 to 1 , where 1 indicates very strong stability (Supplementary Sect. 5).

Lastly, we investigated the predicted clustering strength of the partitioning algorithms using the predict.strength package of Tibshirani et al. [52], which applies the principles of cross-validation, well established in supervised machine learning, to the unsupervised case. Predict.strength performs n-fold random resampling, partitions the resampled population into training and test data folds, and clusters these with varying numbers of clusters through $\mathrm{m}$ iterations. For each iteration, the centroids of training data are applied to test data to compute the proportion of pair-observations falling into the same cluster with the centroids of test data. In the present study, subjects were randomly resampled over 500 iterations and partitioned each time into test and training datasets using two-fold cross-validation. The highest prediction strength over cut off value 0.80 was chosen as the optimal predict.strength value, following published procedures [52] (Supplementary Sect. 6).

The k-means clustering solution survived these validity, stability and generalizability tests and was chosen for further association tests with global and syndromal measures of disease severity, such as the PANSS and SANS total scores, as well as each PANSS and SANS subscales between the identified FTD subgroups. Respective results were reported in Supplementary Sect. 7. To test the specificity of the k-means clustering solution, we run several sanity analyses using items from PANSS and SANS subscales that are not related to FTD and reported the results in Supplementary Sect. 8.
We compared age, educational years, clinical outcomes; Global Social (GF-Social) and Role (GF-Role) Functioning scores, and neuropsychological performances; abstract reasoning, verbal fluency, processing speed, verbal short-term and working memory between FTD subgroups using the Mann-Whitney- $U$ test [32] after checking for normal distribution with the Shapiro-Wilk test [48]. Distributions of sex, site and FTD subgroups, respectively, were compared using the $\chi^{2}$ tests [36] (Tables 1 and 2). All analyses and univariate statistical comparisons were conducted with $\mathrm{R}$ version 3.5.2. We used the False Discovery Rate (FDR) [5] to correct all $P$ values for the multiple comparisons. $P$ values of the Sociodemographic: Age, sex, education, and the number of participants per site, $P$ values of the clinical outcomes and $P$ values of the neurocognition were considered dependent and corrected using FDR. We provided effect sizes calculated with the wilcoxonR function in the $\mathrm{R}$ package rcompanion for each nonparametric statistical comparison (Fig. 1). We tested the FDR-corrected significance of correlations and provided the correlogram that is a graphical representation of the correlation matrix of all included variables. (Fig. 2).

3a) Association of the clustering solution with clinical outcomes; social and role functioning

We investigated the association of the selected clustering solution with clinical outcomes by performing betweengroups statistical comparisons of baseline the GF-Social and GF-Role functioning scores including the retrospective assessments of highest functioning levels lifetime as well as past year. $[10,12]$ Trained clinicians assessed the functioning level of each participant and interrater reliability tests were performed regularly to calibrate the Global Functioning scales; GF-Social [Intraclass Correlation (ICC) $=0.945$ and GF-Role $(I C C=0.924)$ ] across study sites [29]. We compared the differences in social and role functioning scores between two clusters with the Mann-Whitney- $U$ test [32] or with the Welch's two-sample $t$-test [54] after checking for normal distribution with the Shapiro-Wilk test [48].

3b) Association of FTD-defined subgroups and neurocognitive performance

We analysed the following neurocognitive domains; the WAIS-III; premorbid verbal intelligence assessing visual processing and abstract reasoning, the $\mathrm{VF}-\mathrm{P} \& \mathrm{~S}$ assessing verbal fluency and processing speed and the ADS-F \& $\mathrm{B}$ assessing verbal short-term memory and verbal working memory from the neurocognitive battery of the PRONIA study (Supplementary Table 1), because previous literature has shown a significant association between these neurocognitive domains and FTD as measured by Thought, Language, 
Table 2 Clinical, functioning and neurocognition differences in individuals with recent-onset psychosis

\begin{tabular}{|c|c|c|c|c|}
\hline \multirow[t]{2}{*}{ Characteristics } & \multicolumn{4}{|c|}{ Formal thought disorder related symptom severity } \\
\hline & Low & High & $p$ value & $p_{\text {fdr }}$ value \\
\hline \multicolumn{5}{|l|}{$\begin{array}{l}\text { Global functioning } \\
\text { Social scale rated at baseline }\end{array}$} \\
\hline Highest lifetime score, median & 8 & 8 & $<0.001$ & $<0.001$ \\
\hline Highest score in past year, median & 7 & 6 & $<0.001$ & $<0.001$ \\
\hline Lowest score in past year, median & 5 & 5 & 0.005 & 0.005 \\
\hline Current score, median & 6 & 5 & $<0.001$ & $<0.001$ \\
\hline \multicolumn{5}{|l|}{$\begin{array}{l}\text { Global functioning } \\
\text { Role scale rated at baseline }\end{array}$} \\
\hline Highest lifetime score, median & 8 & 8 & $<0.001$ & $<0.001$ \\
\hline Highest score in past year, median & 7 & 6 & $<0.001$ & $<0.001$ \\
\hline Lowest score in past year, median & 5 & 4 & 0.001 & 0.002 \\
\hline Current score, median & 6 & 5 & $<0.001$ & $<0.001$ \\
\hline \multicolumn{5}{|l|}{ Neurocognition at baseline } \\
\hline WAIS_-premorbid verbal intelligence, median & 10 & 9 & 0.001 & 0.002 \\
\hline WAIS-Matrices, median & 10 & 9 & 0.008 & 0.010 \\
\hline Phonological Verbal Fluency, median & 13 & 11 & $<0.001$ & $<0.001$ \\
\hline Semantic Verbal Fluency, median & 21 & 16 & $<0.001$ & $<0.001$ \\
\hline Forward Digit Span, median & 9 & 8 & $<0.001$ & 0.002 \\
\hline Backward Digit Span, median & 6 & 6 & 0.015 & 0.015 \\
\hline
\end{tabular}

and Communication (TLC) [1] and TALD [28] scale [38, 41, $42,51,53]$. Welch's two-sample $t$-test [55] or Mann-Whitney- $U$ test [32] (based on the results of the Shapiro-Wilk test [48]) were used to assess differences between FTD subgroups in their neurocognitive performances.

We ran several sanity analyses with the following aims: (1) to test whether our clustering solution was associated with global and syndromal disease severity, (2) to investigate the interaction between FTD-related symptoms, (3) to corroborate the specificity of our clustering solution with FTD-related symptoms, (4) to exclude that the clustering algorithms provide us with a solution more prone to detect negative symptom pattern and (5) to test whether our clustering protocol recognizes a positive symptom pattern, and reported results in the Supplementary Sects. 7 and 8 in details. These sanity analyses showed us that our FTDdriven clustering solution was clinically valid and specific to FTD-related symptom severity. Analyses with non-FTD items from PANSS and SANS as well as their subscale showed less stable and less generalizable clustering solutions than the FTD-driven clustering solution.

\section{Results}

Our multi-step clustering analyses identified a k-means algorithm-based two-cluster solution as the most stable and generalizable stratification approach. This approach delineated two FTD subgroups, FTD-High and FTD-Low $(n=75$ vs. 204) in our ROP patient cohort (Fig. 1). The clustering solution was significantly informed by (1) observed positive FTD as measured by conceptual disorganization (PANSS P2) $\left(\right.$ median $=4$ vs. $\left.1, p_{\text {fdr }}<0.001, r=0.488\right)$ and poverty of content of speech (SANS 10) (median $=3$ vs. $0, p_{\text {fdr }}<0.001$, $\mathrm{r}=0.712$ ), and (2) observed negative FTD as measured by difficulty in abstract thinking (PANSS N5) (median $=3$ vs. 1 , $p_{\text {fdr }}<0.001, r=0.503$ ), increased latency of response (SANS 12) $\left(\right.$ median $=3$ vs. $\left.0, p_{\mathrm{fdr}}<0.001, \mathrm{r}=0.653\right)$ and poverty of speech $($ SANS 9$)\left(\right.$ median $=2$ vs. $\left.0, p_{\text {fdr }}<0.001, r=0.611\right)$ (Fig. 1).

As reported in Table 1, we observed no significant interaction of FTD-informed subgroups with sex $\left(\chi^{2}=0.7053\right.$, $p_{\mathrm{fdr}}=0.401$, phi $\left.=0.050\right)$ and site $\left(\chi^{2}=16.452, p_{\mathrm{fdr}}=0.073\right.$, phi $=0.243$ ). Furthermore, the distribution of missing data did not show significant differences between FTD subgroups (Supplementary Table 5). At baseline, the FTD-High group was younger than the FTD-Low group (median $=23$ vs. 24 , $p_{\mathrm{fdr}}=0.022, r=0.153$ ) in group level comparison (Supplementary Fig. 5). The two groups also differed in their education level: with fewer years in education in FTD-High group than in FTD-Low group (median $=12$ vs. $14, p_{\mathrm{fdr}}=0.002$, $r=0.209)$. Furthermore, education years and age were positively correlated in each subgroup: FTD-High $(p<0.001$, $r=0.51)$ and FTD-Low $(p<0.001, r=0.36)$.

Cluster assignment was not influenced by global disease severity as measured by PANSS total score at baseline $\left(p_{\mathrm{fdr}}=0.779, r=0.017\right)$ but was associated with more pronounced negative symptoms as measured by SANS total 
A

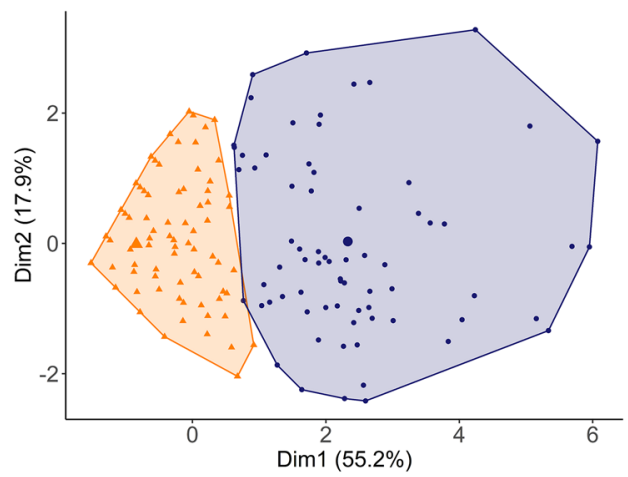

B

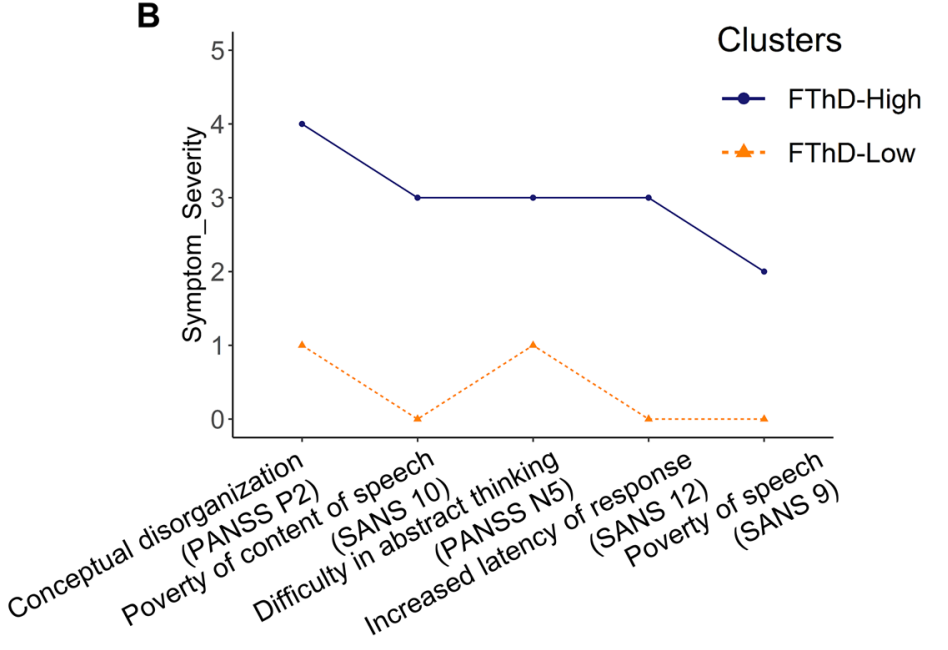

C

Fig. 1 The Psychopathological Comparison of FTD subgroups. A Represents the results of the principal component analysis in twodimensional space, $\mathbf{B}$ the difference between medians of FTD-related symptom severity, $\mathbf{C}$ the distributions of each FTD-related symptom and their statistical comparisons with Wilcoxon rank-sum test. Statistical significances are shown $* * * p_{\mathrm{fdr}}<0.001$
A FThD-High

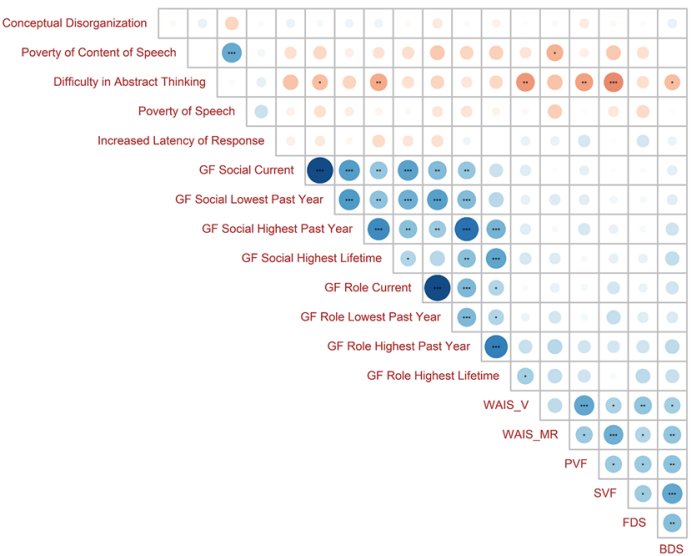

Fig. 2 Correlogram among FTD-related symptoms, functioning and neurocognition; A FTD-High B FTD-Low. Different colours: red $=$ negative or blue $=$ positive represent the direction of correla-
B FThD-Low

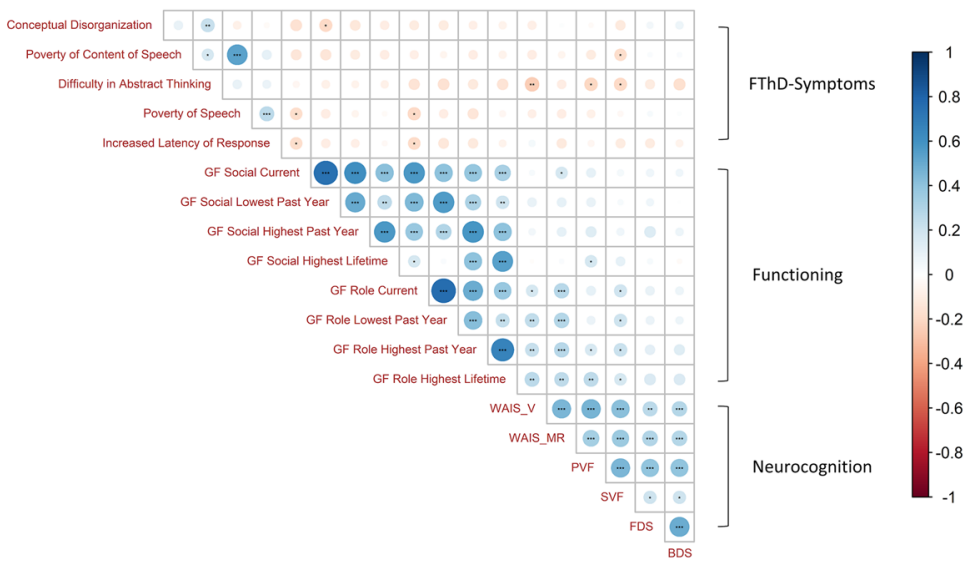

tions, different size of the circles represents the strength of the correlations. Statistical Significances are shown; ${ }^{*} p_{\mathrm{fdr}}<0.05,{ }^{*} p_{\mathrm{fdr}}<0.01$, $* * * p_{\mathrm{fdr}}<0.001$ 
score at baseline $\left(p_{\mathrm{fdr}}<0.001, r=-0.551\right)$ in the high FTD subgroup (Supplementary Table 6).

\section{Comparison of functioning between FTD subgroups}

FTD subgroups differed significantly in the GF-Social and GF-Role instruments (Table 2 and Fig. 3). The GF-Social scores were lower in the FTD-High compared to the FTDLow group in the highest lifetime $\left(p_{\mathrm{fdr}}<0.001, r=-0.216\right)$, past year $\left(p_{\mathrm{fdr}}<0.001, r=-0.219\right)$ and baseline variables $\left(p_{\mathrm{fdr}}<0.001, r=-0.269\right)$. Similarly, GF-Role scores were lower in the FTD-High vs. FTD-Low group in the highest lifetime $\left(p_{\mathrm{fdr}}<0.001, r=-0.229\right)$, past year $\left(p_{\mathrm{fdr}}=0.001\right.$, $r=-0.242)$ and baseline variables $\left(p_{\mathrm{fdr}}<0.001\right.$, $r=-0.259$ ).

\section{Comparison of neurocognitive performance between FTD subgroups}

Comparisons of neurocognitive measures between FTD subgroups showed significant differences in verbal and semantic fluency, verbal short-term memory and abstract reasoning (Table 2). The WAIS-Vocabulary $\left(p_{\mathrm{fdr}}=0.002, r=-0.200\right)$ and WAIS-Matrices ( $\left.p_{\mathrm{fdr}}=0.010, r=-0.166\right)$ scores were lower in the FTD-High group than in the FTD-Low group. We found a similar pattern of results in the phonological verbal fluency ( $p_{\mathrm{fdr}}<0.001, r=-0.235$ ) and semantic fluency $\left(p_{\mathrm{fdr}}<0.001, r=-0.326\right)$ scores, as well as in the forward $\left(p_{\mathrm{fdr}}=0.002, r=-0.204\right)$ and backward $\left(p_{\mathrm{fdr}}=0.015\right.$, $r=-0.151$ ) digit span scores, i.e., FTD-High individuals always performed worse than FTD-Low group in these neurocognitive domains.

\section{Correlation analyses of FTD-related symptoms, functioning and neurocognition}

The correlation analyses in the FTD-High subgroup revealed that "difficulty in abstract thinking" correlated negatively with functioning GF-Social lowest in past year: $\left(p_{\mathrm{fdr}}=0.038, r=-0.30\right)$; GF-Social highest in lifetime: $\left.\left(p_{\mathrm{fdr}}=0.006, r=-0.37\right)\right)$, and neurocognitive domains (WAIS-Vocabulary: $\left(p_{\mathrm{fdr}}=0.002, r=-0.43\right.$ ); backward digit span: ( $\left.p_{\mathrm{fdr}}=0.042, r=-0.30\right)$; phonological verbal fluency: $\left(p_{\mathrm{fdr}}=0.003, r=-0.40\right)$; and semantic fluency: $\left(p_{\mathrm{fdr}}<0.001\right.$, $r=-0.48)$ ). Poverty of content of speech item was significantly associated with WAIS-Matrices $\left(p_{\mathrm{fdr}}=0.016\right.$, $r=-0.35$ ) in the FTD-High subgroup. The correlations between other FTD-related symptoms and other variables in functioning or neurocognition were either non-significant or significant with a small effect size $(r<0.30)$ in both subgroups (Fig. 2).

\section{Discussion}

In this multisite naturalistic study, we addressed for the first time the psychopathological heterogeneity of patients with recent-onset psychosis from the perspective of the core syndrome of FTD with unsupervised machine learning algorithms. Furthermore, we investigated the broader quantitative associations between FTD, neurocognitive performance and level of functioning in the early stage of psychosis with group-level statistical comparisons. Our multi-step clustering analysis yielded a solution with two FTD subgroups as the most optimal stratification scheme after running the following four checkpoints; (i) validity, (ii) re-evaluation of validity results and unbiased determination of the winning algorithm, (iii) stability test and (iv) generalizability test for the best clustering solution. In summary, the winning clustering solution revealed two stable subgroups of patients with high and low severity of FTD, which were independent of global disease severity in this early stage of the disease. The FTD-High subgroup showed significant impairment of all functional domains and significantly lower neurocognitive performance in verbal and semantic fluency, short-term verbal memory and abstract thinking. Moreover, the median age at baseline was one year less in both sexes in the FTDHigh subgroup and an earlier differentiating peak of male distribution was observed in the FTD-High subgroup in the late adolescence and early adulthood. (Supplementary Fig. 5) This is in keeping with previous clinical observations of worse prognostic long-term outcomes in males. [19, 30]

Our results are also in line with the previous literature showing that thought disorders are negatively associated
Fig. 3 The Comparison of functioning levels in social and role functioning domains. Statistical comparisons are conducted with the Welch two-sample or the Mann-Whitney- $U$ tests based on the distribution of the data. Statistical Significances are shown; $* * * p_{\mathrm{fdr}}<0.001$
A

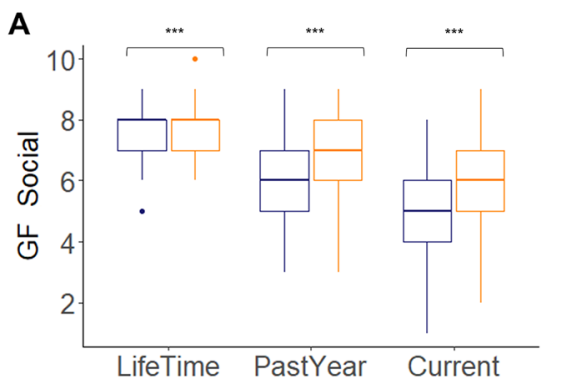

B

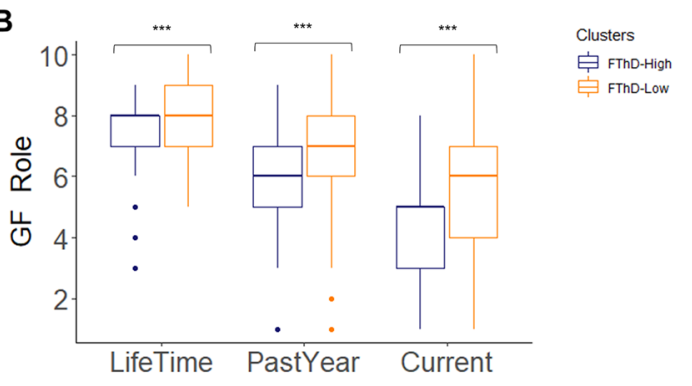


with role functioning [20], and thought and communication disturbances are related to poorer social and role functioning levels [4]. Retrospective clinical assessments at study inclusion indicated that FTD subgroups started to deviate in symptom and disability measures as well as in social and role functioning already in the year prior to the study. The more specific assessment of social and role functioning differences between FTD subgroups extended this observation to the lifetime scale that may point to FTD subtypes being as a sensitive prognostic marker for a later manifestation of psychosis. $[4,18,56]$ These findings may represent a starting point for further investigation of these alterations that may help in identify an early diagnostic and interventional window in which interventions focusing on FTD-related impairments may be beneficial in improving functioning during the early stages of psychosis.

Furthermore, we found that FTD stratification was associated with reduced verbal and semantic fluency, and impairments in short-term verbal memory and abstract thinking. This association between FTD and neurocognitive performance in semantic processing, executive functioning, abstract thinking adds to the previous literature. [38, 41, 42, $50,52]$ We may speculate that the lower neurocognitive performance of FTD-High group could be seen from a causal consequential perspective and may drive the impaired social and occupational functioning in this subgroup requiring regular and frequent follow-up examinations. [40, 57] However, further studies addressing this interrelation between FTD and neurocognitive performance are warranted to validate this speculation. Moreover, future studies should be conducted to understand whether the relationship between cluster assignment and neurocognition is moderated or mediated by the severity of negative symptoms.

\section{Limitations}

A validated specific scale to assess FTD and SAPS (Scale for the Assessment of Positive Symptoms) are missing in the presented study. We assessed a restricted part of FTD spectrum with the overlapping symptoms from PANSS and SANS that mapped on to the TALD. This reduces the complexity of the possible interpretations of our results. Therefore, our findings should be considered as preliminary, and further studies employing a more thorough investigation of FTD manifestations through much more assessments (i.e., TALD, SAPS) are warranted to understand the degree of replicability of our findings. Moreover, the presented study did not differ affective and non-affective psychosis. Another limitation is the cross-sectional nature of the analysed data from the baseline examinations, which significantly limits any causal speculation. Lastly, a missing external validation sample restricts the generalizability of these clustering solutions. Further studies applying presented stratification scheme at the single patient level in an external sample are neededQuery.

\section{Conclusions}

The presented multi-step clustering study demonstrated subgroups of patients with distinct clinical presentations of FTD who may have divergent preventive and therapeutic needs related to differential FTD severity. Our findings elucidate how unsupervised machine learning techniques may provide novel insight about the associations between psychopathology, neurocognition and functioning.

In summary, our findings suggest that FTD may be a relevant marker of illness severity in the early psychosis pathway. The reciprocal associations between FTD, functional, and neuropsychological phenotypes of psychosis emphasize the importance of specific treatment pathways for people with more severe FTD. Furthermore, they highlight how FTD may potentially represent a target variable for individualized psycho-, socio-, logotherapeutic interventions aimed at improving neurocognition abilities and personal functioning. Prospective studies should further test this promising perspective.

Supplementary Information The online version contains supplementary material available at https://doi.org/10.1007/s00406-021-01327-y.

Acknowledgements We thank all subjects for their participation in the study. Oemer Faruk Oeztuerk and David Popovic are supported by International Max Planck Research School for Translational Psychiatry in the frame of Clinician Scientist Program funded by the ElseKröner-Fresenius-Stiftung. Linda A. Antonucci's salary is funded by the Structural European Funding of the Italian Minister of Education (Attraction and International Mobility-AIM — action, grant agreement No 1859959).

Funding Open Access funding enabled and organized by Projekt DEAL.

Open Access This article is licensed under a Creative Commons Attribution 4.0 International License, which permits use, sharing, adaptation, distribution and reproduction in any medium or format, as long as you give appropriate credit to the original author(s) and the source, provide a link to the Creative Commons licence, and indicate if changes were made. The images or other third party material in this article are included in the article's Creative Commons licence, unless indicated otherwise in a credit line to the material. If material is not included in the article's Creative Commons licence and your intended use is not permitted by statutory regulation or exceeds the permitted use, you will need to obtain permission directly from the copyright holder. To view a copy of this licence, visit http://creativecommons.org/licenses/by/4.0/.

\section{References}

1. Andreasen NC (1986) Scale for the assessment of thought, language, and communication (TLC). Schizophr Bull 12(3):473-482 
2. Andreasen NC (1989) The scale for the assessment of negative symptoms (SANS). Br J Psychiatry 155:49-52

3. Andreasen NC, Grove WM (1986) Thought, language, and communication in schizophrenia: diagnosis and prognosis. Schizophr Bull 12(3):348-359

4. Bearden CE, Wu KN, Caplan R, Cannon TD (2011) Thought disorder and communication deviance as predictors of outcome in youth at clinical high risk for psychosis. J Am Acad Child Adolesc Psychiatry 50(7):669-680

5. Benjamini Y, Hochberg Y (1995) Controlling the false discovery rate: a practical and powerful approach to multiple testing. J Roy Stat Soc Ser B (Methodol) 57(1):289-300

6. Burock G, Pihur V, Datta S, Datta S (2008) clValid: an R package for cluster validation. J Stat Softw. https://doi.org/10.18637/jss. v025.i04

7. Bzdok D, Meyer-Lindenberg A (2018) Machine learning for precision psychiatry: opportunities and challenges. Biol Psychiatry Cogn Neurosci Neuroimaging 3(3):223-230

8. Caissie SL, Liddle PF, Ngan ETC, Quested DJ, Bates AT, White R, Weg R, Anderson CM (2002) Thought and Language Index: an instrument for assessing thought and language in schizophrenia. Br J Psychiatry 181(04):326-330

9. Caliński T, Harabasz J (1974) A dendrite method for cluster analysis. Commun Stat Theor Methods 3(1):1-27

10. Carrión RE, McLaughlin D, Goldberg TE, Auther AM, Olsen RH, Olvet DM, Correll CU, Cornblatt BA (2013) Prediction of functional outcome in individuals at clinical high risk for psychosis. JAMA Psychiat 70(11):1133-1142

11. Charrad M, Ghazzali N, Boiteau V, Niknafs A (2014) NbClust: an $\mathrm{R}$ package for determining the relevant number of clusters in a data set. J Stat Softw 61(1):1-36. https://doi.org/10.18637/jss. v061.i06

12. Cornblatt BA, Auther AM, Niendam T, Smith CW, Zinberg J, Bearden CE, Cannon TD (2007) Preliminary findings for two new measures of social and role functioning in the prodromal phase of schizophrenia. Schizophr Bull 33(3):688-702

13. Davies DL, Bouldin DW (1979) A cluster separation measure. IEEE Trans Pattern Anal Mach Intell 2:224-227

14. Docherty NM, DeRosa M, Andreasen NC (1996) Communication disturbance in schizophrenia and mania. Arch Gen Psychiatry $53: 358$

15. Dwyer DB, Falkai P, Koutsouleris N (2018) Machine learning approaches for clinical psychology and psychiatry. Annu Rev Clin Psychol 14(1):91

16. Falkai P, Gaebel W, Weinmann S, Wobrock T (2006) DGPPN (Hrsg.) S3-Praxisleitlinien in Psychiatrie und Psychotherapie Band 1: Behandlungsleitlinie Schizophrenie

17. Gaebel W, Zielasek J (2015) Focus on psychosis. Dialogues Clin Neurosci 17(1):9-18. https://doi.org/10.31887/DCNS.2015.17.1/ wgaebel

18. Gooding DC, Coleman MJ, Roberts SA, Shenton ME, Levy DL, Erlenmeyer-Kimling L (2012) Thought disorder in offspring of schizophrenic parents: findings from the New York High-Risk Project. Schizophr Bull 38(2):263-271

19. Hafner H, Maurer K, Loffler W, Riecher-Rossler A (1993) The influence of age and sex on the onset of early course of schizophrenia. Br J Psychiatry 162:80-86. https://doi.org/10.1192/bjp. 162.1.80

20. Harrow M, Marengo JT (1986) Schizophrenic thought disorder at followup : its persistence and prognostic significance. Schizophr Bull 12(3):373

21. Hart M, Lewine RRJ (2017) Rethinking thought disorder. Schizophr Bull 43(3):514-522

22. Hou C-L, Xiang Y-T, Wang Z-L, Everall I, Tang Y, Yang C, Xu M-Z, Correll CU, Jia F-J (2016) Cognitive functioning in individuals at ultra-high risk for psychosis, first-degree relatives of patients with psychosis and patients with first-episode schizophrenia. Schizophr Res 174(1-3):71-76

23. Iniesta R, Stahl D, McGuffin P (2016) Machine learning, statistical learning and the future of biological research in psychiatry. Psychol Med 46(12):2455-2465

24. Insel TR, Cuthbert BN (2015) Brain disorders? Precisely. Science 348(6234):499-500

25. Jerónimo J, Queirós T, Cheniaux E, Telles-Correia D (2018) Formal thought disorders-historical roots. Front Psychiatry 9(November): $1-5$

26. Kay SR, Fiszbeln A, Opler LA (1987) The positive and negative syndrome scale (panss) manual. Schizophrenia Bull 13(2):261

27. Kircher T, Bröhl H, Meier F, Engelen J (2018) Formal thought disorders: from phenomenology to neurobiology. Lancet Psychiatry $5(6): 515-526$

28. Kircher T, Krug A, Stratmann M, Ghazi S, Schales C, Frauenheim M, Turner L, Fährmann P, Hornig T, Katzev M, Grosvald M, Müller-Isberner R, Nagels A (2014) A rating scale for the assessment of objective and subjective formal thought and language disorder (TALD). Schizophr Res 160(1-3):216-221

29. Koutsouleris N, Kambeitz-Ilankovic L, Ruhrmann S, Rosen M, Ruef A, Dwyer DB, Paolini M, Chisholm K, Kambeitz J, Haidl T (2018) Prediction models of functional outcomes for individuals in the clinical high-risk state for psychosis or with recent-onset depression: a multimodal, multisite machine learning analysis. JAMA Psychiat 75(11):1156-1172

30. Labad X, Cobo J, Usall J, Ochoa S, Kulkarni J (2012) Gender differences in schizophrenia and first-episode psychosis: a comprehensive literature review. Schizophr Res Treat 2012:1-9

31. Lord E, Willems M, Lapointe FJ, Makarenkov V (2017) Using the stability of objects to determine the number of clusters in datasets. Inf Sci 393:29-46

32. Mann HB, Whitney DR (1947) On a test of whether one of two random variables is stochastically larger than the other. Ann Math Statist 18(1):50-60

33. Marder SR, Galderisi S (2017) The current conceptualization of negative symptoms in schizophrenia. World Psychiatry $16: 14-24$

34. Marengo JT, Harrow M (1987) Schizophrenic thought disorder at follow-up. Arch Gen Psychiatry 44:651

35. Marquand AF, Wolfers T, Mennes M, Buitelaar J, Beckmann CF (2016) Beyond lumping and splitting: a review of computational approaches for stratifying psychiatric disorders. Biol Psychiatry Cogn Neurosci Neuroimaging 1(5):433-447

36. McHugh ML (2013) The chi-square test of independence. Biochemia Medica 23(2):143-149

37. Michel C, Ruhrmann S, Schimmelmann BG, Klosterkötter J, Schultze-Lutter F (2018) Course of clinical high-risk states for psychosis beyond conversion. Eur Arch Psychiatry Clin Neurosci 268(1):39-48

38. Nagels A, Fährmann P, Stratmann M, Ghazi S, Schales C, Frauenheim M, Turner L, Hornig T, Katzev M, Müller-Isberner R, Grosvald M, Krug A, Kircher T (2016) Distinct neuropsychological correlates in positive and negative formal thought disorder syndromes: the thought and language disorder scale in endogenous psychoses. Neuropsychobiology 73(3):139-147

39. Oeztuerk OF, Pigoni A, Antonucci LA, Koutsouleris N (2021) Association between formal thought disorders, neurocognition and functioning in the early stages of psychosis: a systematic review of the last half-century studies. Eur Arch Psychiatry Clin Neurosci 1-13. https://doi.org/10.1007/s00406-021-01295-3

40. Racenstein JM, Penn D, Harrow M, Schleser R (1999) Thought disorder and psychosocial functioning in schizophrenia: the concurrent and predictive relationships. J Nerv Ment Dis 187(5):281-289 
41. Remberk B, Namysłowska I, Rybakowski F (2012) Clinical and cognitive correlates of formal thought disorder in early onset schizophrenia. Activitas Nervosa Superior Rediviva 54(2):68-76

42. Remberk B, Namysłowska I, Rybakowski F (2012) Cognitive impairment and formal thought disorders in parents of early-onset schizophrenia patients. Neuropsychobiology 65(4):206-215

43. Roche E, Creed L, Macmahon D, Brennan D, Clarke M (2015) The epidemiology and associated phenomenology of formal thought disorder: a systematic review. Schizophr Bull 41(4):951-962

44. Roche E, Lyne J, O’Donoghue B, Segurado R, Behan C, Renwick L, Fanning F, Madigan K, Clarke M (2016) The prognostic value of formal thought disorder following first episode psychosis. Schizophr Res 178(1-3):29-34

45. Rodrigues-Amorim D, Rivera-Baltanás T, López M, Spuch C, Olivares JM, Agís-Balboa RC (2017) Schizophrenia: a review of potential biomarkers. J Psychiatr Res 93:37-49

46. Rousseeuw PJ (1987) Silhouettes: a graphical aid to the interpretation and validation of cluster analysis. J Comput Appl Math 20:53-65

47. Schultze-Lutter F, Michel C, Schmidt SJ, Schimmelmann BG, Maric NP, Salokangas RKR, Riecher-Rössler A, van der Gaag M, Nordentoft M, Raballo A (2015) EPA guidance on the early detection of clinical high risk states of psychoses. Eur Psychiatry 30(3):405-416

48. Shapiro SS, Wilk MB (1965) An analysis of variance test for normality (complete samples). Biometrika 52(3/4):591-611

49. Sigaudo M, Crivelli B, Castagna F, Giugiario M, Mingrone C, Montemagni C, Rocca G, Rocca P (2014) Quality of life in stable schizophrenia: The relative contributions of disorganization and cognitive dysfunction. Schizophr Res 153(1-3):196-203

50. Stouten LH, Veling W, Laan W, van der Helm M, van der Gaag M (2017) Psychosocial functioning in first-episode psychosis and associations with neurocognition, social cognition, psychotic and affective symptoms. Early Interv Psychiatry 11(1):23-36

51. Tan EJ, Rossell SL (2014) Building a neurocognitive profile of thought disorder in schizophrenia using a standardized test battery. Schizophr Res 152(1):242-245

52. Tibshirani R, Walther G (2005) Cluster validation by prediction strength. J Comput Graph Stat 14(3):511-528

53. Ventura J, Thames AD, Wood RC, Guzik LH, Hellemann GS (2010) Disorganization and reality distorsion in schizophrenia: a meta-analysis of the relationship between positive symptoms and neurocognitive deficits. Schizophr Res 121:1-14

54. Walesiak M, Dudek A (2010) Finding groups in ordinal data: an examination of some clustering procedures. Classification as a Tool for Research. Springer, pp 185-192

55. Welch BL (1947) The generalization of students' problem when several different population variances are involved. Biometrika 34(1/2):28-35

56. Wilcox J, Winokur G, Tsuang M (2012) Predictive value of thought disorder in new-onset psychosis. Compr Psychiatry 53(6):674-678

57. Yalınçetin B, Ulaş H, Var L, Binbay T, Akdede BB, Alptekin K (2016) Relation of formal thought disorder to symptomatic remission and social functioning in schizophrenia. Compr Psychiatry 70:98-104

\section{Authors and Affiliations}

\section{Oemer Faruk Oeztuerk ${ }^{1,2,3}$ - Alessandro Pigoni ${ }^{4} \cdot$ Julian Wenzel $^{5} \cdot$ Shalaila S. Haas $^{6} \cdot$ David Popovic $^{1,2} \cdot$ Anne Ruef $^{1}$. Dominic B. Dwyer ${ }^{1} \cdot$ Lana Kambeitz-Ilankovic $^{5} \cdot$ Stephan Ruhrmann $^{5} \cdot$ Katharine Chisholm $^{7} \cdot$ Paris Lalousis $^{8}$. Sian Lowri Griffiths ${ }^{8}$. Theresa Lichtenstein ${ }^{5} \cdot$ Marlene Rosen $^{5}$. Joseph Kambeitz ${ }^{5}$. Frauke Schultze-Lutter ${ }^{9}$. Peter Liddle ${ }^{10} \cdot$ Rachel Upthegrove $^{7,8} \cdot$ Raimo K. R. Salokangas $^{11} \cdot$ Christos Pantelis $^{12,13}$. Eva Meisenzahl ${ }^{9}$. Stephen J. Wood ${ }^{14,15,16}$. Paolo Brambilla ${ }^{17}$. Stefan Borgwardt ${ }^{18}$. Peter Falkai ${ }^{1}$. Linda A. Antonucci ${ }^{1,19,20}$. Nikolaos Koutsouleris ${ }^{1,3,21}$. the PRONIA Consortium}

1 Department of Psychiatry and Psychotherapy, LudwigMaximilian-University Munich, Nussbaumstr. 7, 80336 Munich, Germany

2 International Max Planck Research School for Translational Psychiatry, Munich, Germany

3 Max Planck Institute for Psychiatry, Munich, Germany

4 MoMiLab Research Unit, IMT School for Advanced Studies Lucca, Lucca, Italy

5 Department of Psychiatry and Psychotherapy, Faculty of Medicine and University Hospital of Cologne, University of Cologne, Cologne, Germany

6 Department of Psychiatry, Icahn School of Medicine at Mount Sinai, New York, USA

7 School of Psychology, Aston University, Birmingham, UK

8 Institute for Mental Health, University of Birmingham, Birmingham, UK

9 Department of Psychiatry and Psychotherapy, Medical Faculty, Heinrich-Heine University, Düsseldorf, Germany
10 Division of Psychiatry and Applied Psychology, Institute of Mental Health, University of Nottingham, Nottingham, UK

11 Department of Psychiatry, University of Turku, Turku, Finland

12 Melbourne Neuropsychiatry Centre, University of Melbourne, Melbourne, Australia

13 Melbourne Health, Melbourne, Australia

14 School of Psychology, University of Birmingham, Birmingham, UK

15 Orygen, The National Centre of Excellence for Youth Mental Health, Melbourne, Australia

16 Centre for Youth Mental Health, University of Melbourne, Melbourne, Australia

17 Department of Neurosciences and Mental Health, Fondazione IRCCS Ca' Granda Ospedale Maggiore Policlinico, University of Milan, Milan, Italy

18 Department of Psychiatry, University Psychiatric Clinic, Psychiatric University Hospital, University of Basel, Basel, Switzerland 
19 Department of Basic Medical Sciences, Neuroscience and Sense Organs, University of Bari "Aldo Moro", Bari, Italy

20 Department of Education, Psychology and Communication Science, University of Bari “Aldo Moro”, Bari, Italy
21 Department of Psychosis Studies, Institute of Psychiatry, Psychology and Neuroscience, King's College London, London, UK 